Chapter 9

\title{
How to Distinguish the Mixture of Two D-wave States from Pure D-wave State of HTSC
}

\author{
Peter Brusov and Tatiana Filatova \\ Additional information is available at the end of the chapter \\ http://dx.doi.org/10.5772/59180
}

\section{Introduction}

The chapter is devoted to the very important problem of the actual symmetry of the order parameter in cuprates, and, more generally, in unconventional superconductors. We adopt the concept of the mixed-order parameter. We calculated collective modes in the $d_{x 2-y 2}+i d_{x y}$ case and found them to differ from those appropriate to the pure situation. The results are useful for both theoretical as well as experimental aspects of the discussed problem.

Several groups have worked in this direction and we can indicate the contributions of Tony Leggett, James Annett, David Pines, Doug Scalapino, and Sasha Balatsky et al. in the theory of the problem and of John Ketterson et al. in its experimental study.

In this chapter we look at collective excitations in unconventional superconductors (USC). This unusual topic was chosen for two reasons: 1) there is no superconductor for which unconventional pairing has been exactly established, while there is some evidence of nontrivial pairing in HFSC and HTSC; 2) the existence of collective excitations in superconductors is questionable.

Within the last couple of decades the situation has drastically changed, collective excitations becoming increasingly important in studies and experiments on USC.

An amplitude mode has been observed in ordinary superconductor films with frequency of order $2 \Delta$. Furthermore, the type of pairing has been established for most superconductors. There is an s-pairing in electron-type HTSC and in ordinary superconductors, a p-pairing in pure ${ }^{3} \mathrm{He} ;{ }^{3} \mathrm{He}$ in aerogel, $\mathrm{Sr}_{2} \mathrm{RuO}_{4}$ (HTSC), $\mathrm{UPt}_{3}$ (HFSC), and d-pairing in hole-type HTSC, organic superconductors, and some HFSC $\left(\mathrm{UPd}_{2} \mathrm{Al}_{3}, \mathrm{CePd}_{2} \mathrm{Si}_{2}, \mathrm{CeIn}_{3}, \mathrm{CeNi}_{2} \mathrm{Ge}_{2}\right.$, etc.).

In 2002 a microwave surface-impedance study of the HFSC $\mathrm{UBe}_{13}$ demonstrated an absorption peak [16]. The frequency-dependence as well as temperature-dependence of this peak scales 
with the BCS gap function $\Delta(\mathrm{T})$. This means that this was the first direct observation of the resonant absorption into a collective mode $(\mathrm{CM})$. The $\mathrm{CM}$ energy turned out to be proportional to the superconducting gap. This was therefore a new page in the study of collective excitations in USC.

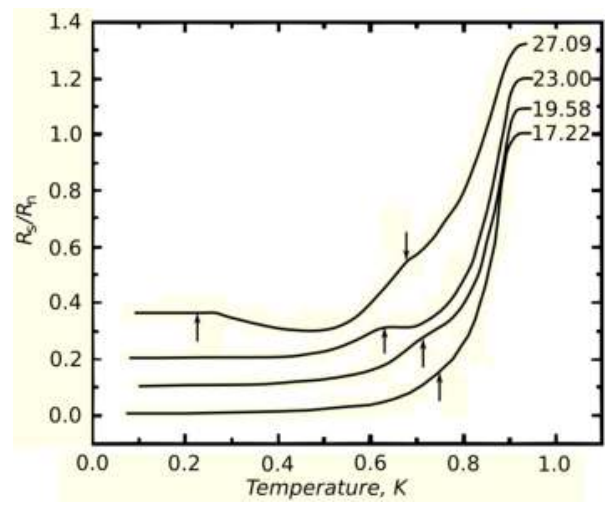

Figure 1. Temperature dependence of surface impedance in heavy-fermion superconductor $\mathrm{UBe}_{13}$ [Ref. 16].

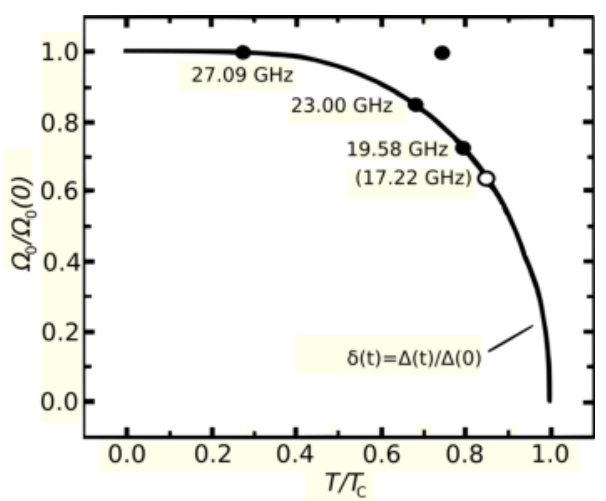

Figure 2. Normalized frequency of collective mode in heavy-fermion superconductor $\mathrm{UBe}_{13}$ [Ref. 16]

\section{Two d-wave states mixture}

As has been shown both experimentally [1] and theoretically, [2,3] in HTSC a mixing of different $\mathrm{d}$-wave states takes place. The collective-mode spectrum in a mixed $d_{x 2-y 2}+i d_{x y}$ state of HTSC was calculated by Paul Brusov and Peter Brusov [4] for the first time. Within the d-pairing model for superconductive and superfluid Fermi-systems (HTSC, HFSC, etc.) earlier created by Brusov and Brusov within the path integration technique, [5-7] they showed that the spectrum in 
mixture $d_{x 2-y 2}+i d_{x y}$ state turns out to be quite different from spectra in both states $d_{x 2-y 2}$ and $d_{x y}$ in spite of the fact that both spectra are identical. $[8,10]$ Thus the ultrasound and/or microwave absorption experiments could be used as the probe of the CM spectrum, allowing the pure $\mathrm{d}$ wave states to be distinguished from the mixture of the two d-wave states [18].

While most scientists believe that there is a $d$-wave pairing in HTSC, there is still an active debate over different ideas concerning mixture of $s$ - and $d$-states, extended s-wave pairing, and mixture of different $d$-states [18]. The main cause of this is the absence of answers to the question of whether there is an exact-zero gap along some chosen lines in momentum space (as in the case of $d_{x 2-y 2}$ ) or an anisotropic gap that remains nonzero everywhere (except maybe at some points). There is no certain answer to this question that has been yielded by existing experiments (tunnelling, etc.) but the answer is quite principled. However, there are some experiments [1] which, suggesting realization in HTSC in a mixed state like $d_{x 2-y 2}+i d_{x y}$, could provide an explanation [3]. The possibility of a mixture of different d-wave states in HTSC has been considered by Annett et al., [2] who came to the conclusion that the most likely state is $d_{x 2-y 2}+i d_{x y} \cdot[18]$

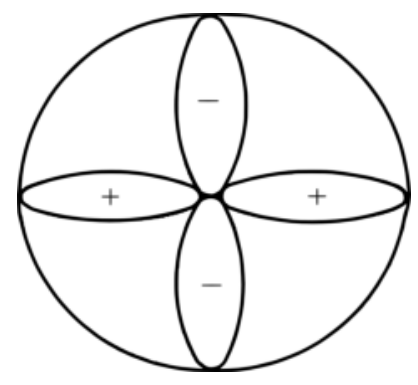

Figure 3. Gap symmetry in pure $d$-wave state $\left(d_{x y}\right.$-state).

One possible way to distinguish pure $d$-states from mixture was suggested by Peter Brusov and Pavel Brusov[4], who considered the mixed $d_{x 2-y 2}+i d_{x y}$ state and calculated the spectrum of collective modes in this state. The comparison of the spectrum of a pure d-wave state of HTSC with the spectrum of the mixed $d_{x 2-y 2}+i d_{x y}$ state shows that they are significantly different. This means that they could be used as the probe of the symmetry of the order parameter in HTSC. [18]

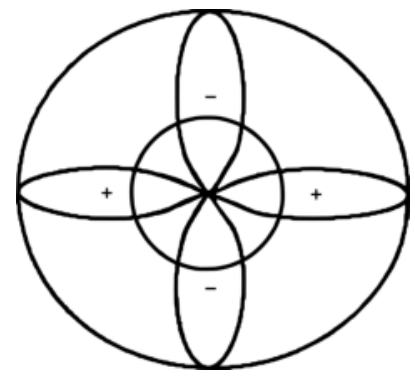

Figure 4. Gap symmetry in the mixed s-wave and d-wave states $\left(s+i d_{x y}\right)$. 


\section{Collective-Modes Spectrum Equations in a Mixed d-Wave State}

\subsection{Mixed d-wave-state model}

Brusov et al.'s [4] study is generalized here for the case of arbitrary $d_{x y}$-state admixture. The mixed $(1-\gamma) d_{x-y}^{2}{ }^{2}+i \gamma d_{x y}$ state in high-temperature superconductors is considered here and a full set of equations for the collective-modes spectrum in mixed d-wave state with arbitrary admixture of $\mathrm{d}_{\mathrm{xy}}$-state is derived.

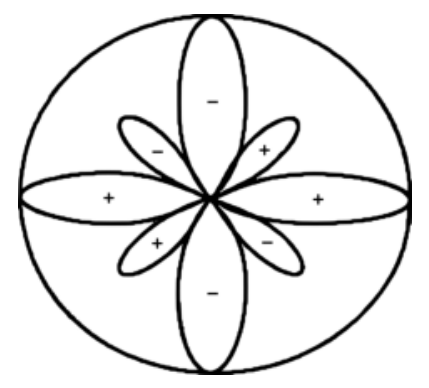

Figure 5. Gap symmetry in the mixed $(1-\gamma) d_{x^{2}-y}^{2}+i \gamma d_{x y}$ state.

We have used the model of $d$-pairing in high-temperature superconductors and HFSC, created by Brusov et al. $[5,11]$

It is described by the effective functional of action:

$$
S_{\text {eff }}=g^{-1} \sum_{p, i, a} c_{i a}^{+}(p) c_{i a}(p)+\frac{1}{2} \ln \operatorname{det} \frac{\hat{M}\left(c_{i a}, c_{i a}^{+}\right)}{\hat{M}\left(c_{i a}^{(0)}, c_{i a}^{(0)+}\right)},
$$

where $c_{i a}^{(0)}$ is the condensate value of Bose-fields $c_{i a}$ (symmetric traceless matrix) and $\hat{M}\left(c_{i a}, c_{i a}^{+}\right)$ is the $4 \times 4$ depending on Bose-fields and parameters of quasi-fermions matrix.

In the case of the $d$-pairing the number of degrees of freedom is equal to 10 . This means that we have five complex canonical variables:

$$
\begin{aligned}
& c_{1}=c_{11}+c_{22}, c_{2}=c_{11}-c_{22}, c_{3}=c_{12}+c_{21}, \\
& c_{4}=c_{13}+c_{31}, c_{5}=c_{23}+c_{32} .
\end{aligned}
$$

The effective action becomes equal to:

$$
S_{\text {eff }}=(2 g)^{-1} \sum_{p, j} c_{j}^{+}(p) c_{j}(p)\left(1+2 \delta_{j 1}\right)+\frac{1}{2} \ln \operatorname{det} \frac{\hat{M}\left(c_{j}^{+}, c_{j}\right)}{\hat{M}\left(c_{j}^{+(0)}, c_{j}^{(0)}\right)},
$$


where

$$
\begin{aligned}
& M_{11}=Z^{-1}[i \omega+\xi-\mu(\mathbf{H} \sigma)] \delta_{p_{1} p_{2}} \\
& M_{22}=Z^{-1}[-i \omega+\xi+\mu(\mathbf{H} \sigma)] \delta_{p_{1} p_{2}} \\
& M_{12}=M_{21}^{*}=(\beta V)^{-1 / 2}\left(\frac{15}{32 \pi}\right)^{1 / 2}\left[c_{1}\left(1-3 \cos ^{2} \theta\right)+\right. \\
& +c_{2} \sin ^{2} \theta \cos 2 \varphi+c_{3} \sin ^{2} \theta \sin 2 \varphi+ \\
& \left.+c_{4} \sin 2 \theta \cos \varphi+c_{5} \sin 2 \theta \sin \varphi\right]
\end{aligned}
$$

here $\boldsymbol{H}$-magnetic field, $\sigma=\left(\sigma_{1}, \sigma_{2}, \sigma_{3}\right)$ - Pauli-matrices, $p=(\mathbf{k}, \omega) ; \omega=(2 n+1) \pi T$ are Fermifrequencies and $x=(\mathbf{x}, \tau), \xi$ is the kinetic energy with respect to Fermi-level, $\mu$-magnetic moment of quasi-fermion.

All the properties of the model superconducting Fermi-system with $d$-pairing are determined by functional (2).

Let us consider the mixed $(1-\gamma) d_{x-y}{ }^{2}+i \gamma d_{x y}$ state of high-temperature superconductors. The order parameter in this state takes the following form:

$$
\Delta_{0}(T)\left[(1-\gamma)\left(\begin{array}{rrr}
1 & 0 & 0 \\
0 & -1 & 0 \\
0 & 0 & 0
\end{array}\right)+i \gamma\left(\begin{array}{lll}
0 & 1 & 0 \\
1 & 0 & 0 \\
0 & 0 & 0
\end{array}\right)\right]
$$

or in canonical variables:

$$
\Delta_{0}(T)\left(0 ;(1-\gamma) \sin ^{2} \theta \cos 2 \varphi ; i \gamma \sin ^{2} \theta \sin 2 \varphi ; 0 ; 0\right)
$$

The gap equation has the following form:

$$
g^{-1}+\frac{\alpha^{2} Z^{2}}{2 \beta V} \sum_{p} \frac{\sin ^{4} \Theta\left[\gamma^{2}+\cos ^{2} 2 \phi(1-2 \gamma)\right]}{\omega^{2}+\xi^{2}+\Delta_{0}^{2} \sin ^{4} \Theta\left[\gamma^{2}+\cos ^{2} 2 \phi(1-2 \gamma)\right]}=0
$$

where

$\Delta_{0}=2 c Z \alpha, \alpha=(15 / 32 \pi)^{1 / 2}$ and gap

$$
\Delta^{2}(T)=\Delta_{0}^{2} \sin ^{4} \Theta\left[\gamma^{2}+\cos ^{2} 2 \phi(1-2 \gamma)\right]
$$


For a limited case of $\gamma=0$, we obtain $d_{x 2-y 2}$ state with order parameter $\left(0 ; \sin ^{2} \theta \cos 2 \phi ; 0 ; 0 ; 0\right)$.

The gap equation in this case has the following form:

$$
g^{-1}+\frac{\alpha^{2} Z^{2}}{2 \beta V} \sum_{p} \frac{\sin ^{4} \Theta \cos ^{2} 2 \phi}{\omega^{2}+\xi^{2}+\Delta_{0}^{2} \sin ^{4} \Theta \cos ^{2} 2 \phi}=0
$$

and gap

$$
\Delta^{2}(T)=\Delta_{0}^{2} \sin ^{4} \Theta \cos ^{2} 2 \phi
$$

For limited case $\gamma=1$ one gets $d_{x y}$-state with order parameter $\Delta_{0}(T)\left(0 ; 0 ; i \sin ^{2} \theta \sin 2 \phi ; 0 ; 0\right)$.

The gap equation has the following form:

$$
g^{-1}+\frac{\alpha^{2} Z^{2}}{2 \beta V} \sum_{p} \frac{\sin ^{4} \Theta \sin ^{2} 2 \phi}{\omega^{2}+\xi^{2}+\Delta_{0}^{2} \sin ^{4} \Theta \sin ^{2} 2 \phi}=0
$$

and gap

$$
\Delta^{2}(T)=\Delta_{0}^{2} \sin ^{4} \Theta \sin ^{2} 2 \phi
$$

Brusov et al.[8] case of equal admixtures of $d_{x 2-y 2}$ - and $d_{x y}$-states in our consideration corresponds to the case $\gamma=1 / 2$. The order parameter takes the following form:

$$
\Delta_{0}(T)\left(0 ; \sin ^{2} \theta \cos 2 \varphi ; i \sin ^{2} \theta \sin 2 \varphi ; 0 ; 0\right)
$$

The gap equation has the following form:

$$
g^{-1}+\frac{\alpha^{2} Z^{2}}{2 \beta V} \sum_{p} \frac{\sin ^{4} \Theta}{\omega^{2}+\xi^{2}+\Delta_{0}^{2} \sin ^{4} \Theta}=0
$$

and gap $\Delta(T)=\Delta_{0}(T) \sin ^{2} \theta$.

\subsection{Equations for collective-modes spectrum in a mixed d-wave state at arbitrary admixture of $d_{x y}$-state}

In the first approximations the spectrum of collective excitations is determined by the quadratic part of $S_{\text {effi }}$ obtained after shift $c_{j} \rightarrow c_{j}+c_{j}{ }^{\circ}$. Here $c_{j}^{o}$ are the condensate values of $c_{j}$ of the following form:[5, 11] 


$$
c_{j}^{0}(p)=(\beta V)^{1 / 2} c \delta_{p 0} b_{j}^{0} \text { and } b_{2}^{0}=2(1-\gamma), \quad b_{3}^{0}=2 i \gamma
$$

with all remaining components of $b_{j}^{0}$ equal to zero.

Excluding terms involving $g^{-1}$ by gap equation, one obtains the following form for the quadratic part of $S_{h}$ :

$$
\begin{aligned}
& S_{H}=\frac{\alpha^{2} Z^{2}}{8 \beta V} \sum_{p} \frac{\left[c^{0} Y^{*}\right]\left[c^{+0} Y\right]}{\omega^{2}+\xi^{2}+\left[c^{0} Y^{*}\right]\left[c^{+0} Y\right]} \sum_{j}(1+2 \delta j 1) c_{j}^{+}(p) c_{j}(p) \\
& +Z^{2} / 4 \beta V \sum_{p 1+p 2=p} \frac{1}{M_{1} M_{2}}\left\{( i \omega _ { 1 } + \xi _ { 1 } ) ( i \omega _ { 2 } + \xi _ { 2 } ) \left(\left[c^{+}(p) \mathrm{Y}\left(p_{2}\right)\right]\right.\right. \\
& \left.\left[c(p) \mathrm{Y}^{*}\left(p_{1}\right)\right]+\left[c^{+}(p) \mathrm{Y}\left(p_{1}\right)\right]\left[c(p) \mathrm{Y}^{*}\left(p_{2}\right)\right]\right)-\Delta^{2}\left[c^{+}(p) \mathrm{Y}\left(-p_{1}\right)\right] \\
& \left.\left.\left[c^{+}(-p) \mathrm{Y}\left(-p_{2}\right)\right]-\Delta^{+2}\left[c(p) \mathrm{Y}^{*}\left(-p_{1}\right)\right]\left[c(-p) \mathrm{Y}^{*}\left(-p_{2}\right)\right]\right]\right\}
\end{aligned}
$$

Here,

$$
\begin{aligned}
& {\left[c \mathrm{Y}^{*}\right]=c_{1}\left(1-3 \cos ^{2} \theta\right)+c_{2} \sin ^{2} \theta \cos 2 \varphi+c_{3} \sin ^{2} \theta \sin 2 \varphi+} \\
& +c_{4} \sin 2 \theta \cos \varphi+c_{5} \sin 2 \theta \sin \varphi .
\end{aligned}
$$

The quadratic form coefficients are proportional to the sums of the Green's functions of quasifermions products. One can go from summation to integration at low temperatures $\left(T_{c}-T \sim T_{c}\right)$ by the following rule:

$$
\frac{1}{\beta V} \sum_{p} \rightarrow \frac{1}{(2 \pi)^{4}} \frac{k_{F}^{2}}{c_{F}} \int d \omega d \xi d \Omega
$$

To evaluate these integrals it is useful to use the Feynman equality:

$$
\begin{aligned}
& {\left[\left(\omega_{1}^{2}+\xi_{1}^{2}+\Delta^{2}\right)\left(\omega_{2}^{2}+\xi_{2}^{2}+\Delta^{2}\right)\right]^{-1}=} \\
& \int d \lambda\left[\alpha\left(\omega_{1}^{2}+\xi_{1}^{2}+\Delta^{2}\right)+(1-\lambda)\left(\omega_{2}^{2}+\xi_{2}^{2}+\Delta^{2}\right)\right]^{-2}
\end{aligned}
$$

It is easy to evaluate the integrals with respect to variables $\omega$ and $\xi$ can also be easy evaluated. Then, they can be evaluated with respect to parameter $\alpha$ and the angular variables.

We obtain the following set of ten equations for the whole spectrum of the collective modes for $(1-\gamma) d_{x-y}^{2}{ }^{2}+i \gamma d_{x y}$ state at arbitrary $\gamma$ after calculating all integrals, except over the angular variables and equating the determinant of the resulting quadratic form to zero: 


$$
\begin{aligned}
& \int_{0}^{1} d x \int d \varphi\left\{\frac{\sqrt{\omega^{2}+4\left(1-x^{2}\right)^{2}\left[\gamma^{2}+\cos ^{2} 2 \phi(1-2 \gamma)\right]}}{\omega} \times\right. \\
& \times \ln \frac{\sqrt{\omega^{2}+4\left(1-x^{2}\right)^{2}\left[\gamma^{2}+\cos ^{2} 2 \phi(1-2 \gamma)\right]}+\omega}{\sqrt{\omega^{2}+4\left(1-x^{2}\right)^{2}\left[\gamma^{2}+\cos ^{2} 2 \phi(1-2 \gamma)\right]}-\omega}\left(1-3 x^{2}\right)^{2}+\left[\left(1-3 x^{2}\right)^{2}-\right. \\
& \left.\left.-\frac{3}{8}\left(\left(1-3 x^{2}\right)^{2}+3\left(1-x^{2}\right)^{2} \cos ^{2} 2 \phi\right)\right] \ln \left(1-x^{2}\right)^{2}\left[\gamma^{2}+\cos ^{2} 2 \phi(1-2 \gamma)\right]\right\}=0 \\
& \int_{0}^{1} d x \int d \varphi\left\{\frac{\omega}{\sqrt{\omega^{2}+4\left(1-x^{2}\right)^{2}\left[\gamma^{2}+\cos ^{2} 2 \phi(1-2 \gamma)\right]}} \cdot\left(1-3 x^{2}\right)^{2} \times\right. \\
& \times \ln \frac{\sqrt{\omega^{2}+4\left(1-x^{2}\right)^{2}\left[\gamma^{2}+\cos ^{2} 2 \phi(1-2 \gamma)\right]}+\omega}{\sqrt{\omega^{2}+4\left(1-x^{2}\right)^{2}\left[\gamma^{2}+\cos ^{2} 2 \phi(1-2 \gamma)\right]}-\omega}+\left[\left(1-3 x^{2}\right)^{2}-\right. \\
& \left.\left.-\frac{3}{8}\left(\left(1-3 x^{2}\right)^{2}+3\left(1-x^{2}\right)^{2} \cos ^{2} 2 \phi\right)\right] \ln \left(1-x^{2}\right)^{2}\left[\gamma^{2}+\cos ^{2} 2 \phi(1-2 \gamma)\right]\right\}=0 \\
& \int_{0}^{1} d x \int d \varphi\left\{\frac{\sqrt{\omega^{2}+4\left(1-x^{2}\right)^{2}\left[\gamma^{2}+\cos ^{2} 2 \phi(1-2 \gamma)\right]}}{\omega} \cdot\left(1-x^{2}\right)^{2} \cos ^{2} 2 \phi \times\right. \\
& \times \ln \frac{\sqrt{\omega^{2}+4\left(1-x^{2}\right)^{2}\left[\gamma^{2}+\cos ^{2} 2 \phi(1-2 \gamma)\right]}+\omega}{\sqrt{\omega^{2}+4\left(1-x^{2}\right)^{2}\left[\gamma^{2}+\cos ^{2} 2 \phi(1-2 \gamma)\right]}-\omega}+\left[\left(1-x^{2}\right)^{2} \cos ^{2} 2 \phi-\right. \\
& \left.\left.-2\left(1-x^{2}\right)^{2} x^{2} \cos ^{2} \phi\right] \ln \left(1-x^{2}\right)^{2}\left[\gamma^{2}+\cos ^{2} 2 \phi(1-2 \gamma)\right]\right\}=0 \\
& \int_{0}^{1} d x \int d \varphi\left\{\frac{\omega}{\sqrt{\omega^{2}+4\left(1-x^{2}\right)^{2}\left[\gamma^{2}+\cos ^{2} 2 \phi(1-2 \gamma)\right]}} \cdot\left(1-x^{2}\right)^{2} \cos ^{2} 2 \phi \times\right. \\
& \times \ln \frac{\sqrt{\omega^{2}+4\left(1-x^{2}\right)^{2}\left[\gamma^{2}+\cos ^{2} 2 \phi(1-2 \gamma)\right]}+\omega}{\sqrt{\omega^{2}+4\left(1-x^{2}\right)^{2}\left[\gamma^{2}+\cos ^{2} 2 \phi(1-2 \gamma)\right]}-\omega}+\left[\left(1-x^{2}\right)^{2} \cos ^{2} 2 \phi-\right. \\
& \left.\left.\left.-2\left(1-x^{2}\right)^{2} x^{2} \cos ^{2} \phi\right)\right] \ln \left(1-x^{2}\right)^{2}\left[\gamma^{2}+\cos ^{2} 2 \phi(1-2 \gamma)\right]\right\}=0 \\
& \int_{0}^{1} d x \int d \varphi\left\{\frac{\sqrt{\omega^{2}+4\left(1-x^{2}\right)^{2}\left[\gamma^{2}+\cos ^{2} 2 \phi(1-2 \gamma)\right]}}{\omega} \cdot 4\left(1-x^{2}\right)^{2} x^{2} \cos ^{2} \phi \times\right. \\
& \times \ln \frac{\sqrt{\omega^{2}+4\left(1-x^{2}\right)^{2}\left[\gamma^{2}+\cos ^{2} 2 \phi(1-2 \gamma)\right]}+\omega}{\sqrt{\omega^{2}+4\left(1-x^{2}\right)^{2}\left[\gamma^{2}+\cos ^{2} 2 \phi(1-2 \gamma)\right]}-\omega}+\left[4\left(1-x^{2}\right)^{2} \times\right. \\
& \left.\left.\times x^{2} \cos ^{2} \phi-2\left(1-x^{2}\right)^{2} x^{2} \cos ^{2} \phi\right] \ln \left(1-x^{2}\right)^{2}\left[\gamma^{2}+\cos ^{2} 2 \phi(1-2 \gamma)\right]\right\}=0
\end{aligned}
$$




$$
\begin{aligned}
& \int_{0}^{1} d x \int d \varphi\left\{\frac{\omega}{\sqrt{\omega^{2}+4\left(1-x^{2}\right)^{2}\left[\gamma^{2}+\cos ^{2} 2 \phi(1-2 \gamma)\right]}} \cdot 4\left(1-x^{2}\right)^{2} x^{2} \cos ^{2} \phi \times\right. \\
& \times \ln \frac{\sqrt{\omega^{2}+4\left(1-x^{2}\right)^{2}\left[\gamma^{2}+\cos ^{2} 2 \phi(1-2 \gamma)\right]}+\omega}{\sqrt{\omega^{2}+4\left(1-x^{2}\right)^{2}\left[\gamma^{2}+\cos ^{2} 2 \phi(1-2 \gamma)\right]}-\omega}+\left[4\left(1-x^{2}\right)^{2} x^{2} \cos ^{2} \phi-\right. \\
& \left.\left.\left.-2\left(1-x^{2}\right)^{2} x^{2} \cos ^{2} \phi\right)\right] \ln \left(1-x^{2}\right)^{2}\left[\gamma^{2}+\cos ^{2} 2 \phi(1-2 \gamma)\right]\right\}=0 \\
& \int_{0}^{1} d x \int d \varphi\left\{\frac{\sqrt{\omega^{2}+4\left(1-x^{2}\right)^{2}\left[\gamma^{2}+\cos ^{2} 2 \phi(1-2 \gamma)\right]}}{\omega} \cdot 4\left(1-x^{2}\right)^{2} x^{2} \sin ^{2} \phi \times\right. \\
& \times \ln \frac{\sqrt{\omega^{2}+4\left(1-x^{2}\right)^{2}\left[\gamma^{2}+\cos ^{2} 2 \phi(1-2 \gamma)\right]}+\omega}{\sqrt{\omega^{2}+4\left(1-x^{2}\right)^{2}\left[\gamma^{2}+\cos ^{2} 2 \phi(1-2 \gamma)\right]}-\omega}+\left[4\left(1-x^{2}\right)^{2} x^{2} \sin ^{2} \phi-\right. \\
& \left.\left.-2\left(1-x^{2}\right)^{2} x^{2} \cos ^{2} \phi\right] \ln \left(1-x^{2}\right)^{2}\left[\gamma^{2}+\cos ^{2} 2 \phi(1-2 \gamma)\right]\right\}=0 \\
& \int_{0}^{1} d x \int d \varphi\left\{\frac{\omega}{\sqrt{\omega^{2}+4\left(1-x^{2}\right)^{2}\left[\gamma^{2}+\cos ^{2} 2 \phi(1-2 \gamma)\right]}} \cdot 4\left(1-x^{2}\right)^{2} x^{2} \sin ^{2} \phi \times\right. \\
& \times \ln \frac{\sqrt{\omega^{2}+4\left(1-x^{2}\right)^{2}\left[\gamma^{2}+\cos ^{2} 2 \phi(1-2 \gamma)\right]}+\omega}{\sqrt{\omega^{2}+4\left(1-x^{2}\right)^{2}\left[\gamma^{2}+\cos ^{2} 2 \phi(1-2 \gamma)\right]}-\omega}+\left[4\left(1-x^{2}\right)^{2} x^{2} \sin ^{2} \phi-\right. \\
& \left.\left.\left.-2\left(1-x^{2}\right)^{2} x^{2} \cos ^{2} \phi\right)\right] \ln \left(1-x^{2}\right)^{2}\left[\gamma^{2}+\cos ^{2} 2 \phi(1-2 \gamma)\right]\right\}=0 \\
& \int_{0}^{1} d x \int d \varphi\left\{\frac{\sqrt{\omega^{2}+4\left(1-x^{2}\right)^{2}\left[\gamma^{2}+\cos ^{2} 2 \phi(1-2 \gamma)\right]}}{\omega} \cdot\left(1-x^{2}\right)^{2} \sin ^{2} \phi \times\right. \\
& \times \ln \frac{\sqrt{\omega^{2}+4\left(1-x^{2}\right)^{2}\left[\gamma^{2}+\cos ^{2} 2 \phi(1-2 \gamma)\right]}+\omega}{\sqrt{\omega^{2}+4\left(1-x^{2}\right)^{2}\left[\gamma^{2}+\cos ^{2} 2 \phi(1-2 \gamma)\right]}-\omega}+\left[\left(1-x^{2}\right)^{2} \sin ^{2} \phi-\right. \\
& \left.\left.-2\left(1-x^{2}\right)^{2} x^{2} \cos ^{2} \phi\right] \ln \left(1-x^{2}\right)^{2}\left[\gamma^{2}+\cos ^{2} 2 \phi(1-2 \gamma)\right]\right\}=0 \\
& \int_{0}^{1} d x \int d \varphi\left\{\frac{\omega}{\sqrt{\omega^{2}+4\left(1-x^{2}\right)^{2}\left[\gamma^{2}+\cos ^{2} 2 \phi(1-2 \gamma)\right]}} \cdot\left(1-x^{2}\right)^{2} \sin ^{2} \phi \times\right. \\
& \times \ln \frac{\sqrt{\omega^{2}+4\left(1-x^{2}\right)^{2}\left[\gamma^{2}+\cos ^{2} 2 \phi(1-2 \gamma)\right]}+\omega}{\sqrt{\omega^{2}+4\left(1-x^{2}\right)^{2}\left[\gamma^{2}+\cos ^{2} 2 \phi(1-2 \gamma)\right]}-\omega}+\left[\left(1-x^{2}\right)^{2} \sin ^{2} \phi-\right. \\
& \left.\left.\left.-2\left(1-x^{2}\right)^{2} x^{2} \cos ^{2} \phi\right)\right] \ln \left(1-x^{2}\right)^{2}\left[\gamma^{2}+\cos ^{2} 2 \phi(1-2 \gamma)\right]\right\}=0 \text {. }
\end{aligned}
$$


We have used the following substitutions: $\cos \theta=x, \omega=\omega / \Delta_{0}$.

The whole spectrum of collective modes in mixed $(1-\gamma) d_{x-y}{ }^{2}+i \gamma d_{x y}$ state of HTSC and arbitrary admixture of $d_{x y}$-state is determined by equations (17). For interpretation of the sound attenuation and microwave absorption data as well as for identification of the type of pairing and order parameter in unconventional superconductors, knowledge of the collective-mode spectrum could be used. They allow, in particular, evaluation of the extent of admixture of a $d_{x y}$-state in a mixed state.

Suppose that the dominant state is $d_{x-y}^{2}{ }^{2}$-state and admixture of $d_{x y}$-state is small, say $5-10 \%$; thus, the most interesting case turns out to be the case of small $\gamma$. One could in this case expand all expressions in powers of small $\gamma$ and obtain the corrections to the spectrum of pure $d_{x-y}^{2}{ }^{2}$ state, as found previously. $[4,5,11]$

\subsection{Equations for collective-modes spectrum in a mixed-d-wave state with equal admixtures of $d_{x-y}^{2}{ }^{2}$ - and $d_{x y}$-states}

Brusov et al. [4] supposed equal admixtures of $d_{x-y}^{2}{ }^{2}$ - and $d_{x y}$-states - in our consideration this corresponds to the case $\gamma=1 / 2$ - and derived the following equations:

$$
\begin{aligned}
& i=1 \\
& \int_{0}^{1} d x \int d \varphi\left\{\frac{\sqrt{\omega^{2}+4 f}}{\omega} \ln \frac{\sqrt{\omega^{2}+4 f}+\omega}{\sqrt{\omega^{2}+4 f}-\omega} g_{1}+\left(g_{1}-\frac{3}{2} f_{1}\right) \ln f\right\}=0 \\
& \int_{0}^{1} d x \int d \varphi\left\{\frac{\omega}{\sqrt{\omega^{2}+4 f}} \ln \frac{\sqrt{\omega^{2}+4 f}+\omega}{\sqrt{\omega^{2}+4 f}-\omega} g_{1}+\left(g_{1}-\frac{3}{2} f_{1}\right) \ln f\right\}=0 \\
& \int_{0}^{i} d x \int d \varphi\left\{\frac{\sqrt{\omega^{2}+4 f}}{\omega} \ln \frac{\sqrt{\omega^{2}+4 f}+\omega}{\sqrt{\omega^{2}+4 f}-\omega} g_{i}+\left(g_{i}-\frac{1}{2} g\right) \ln f\right\}=0 \\
& \int_{0}^{1} d x \int d \varphi\left\{\frac{\omega}{\sqrt{\omega^{2}+4 f}} \ln \frac{\sqrt{\omega^{2}+4 f}+\omega}{\sqrt{\omega^{2}+4 f}-\omega} g_{i}+\left(g_{i}-\frac{1}{2} g\right) \ln f\right\}=0 .
\end{aligned}
$$

Here,

$$
\begin{aligned}
& g_{1}=\left(1-3 x^{2}\right)^{2} ; \\
& g_{2}=\left(1-x^{2}\right)^{2} \cos ^{2} 2 \varphi ; g_{3}=g=4\left(1-x^{2}\right) x^{2} \cos ^{2} \varphi ; \\
& g_{4}=4\left(1-x^{2}\right) x^{2} \sin ^{2} \varphi ; g_{5}=\left(1-x^{2}\right)^{2} \sin ^{2} \varphi, \\
& f_{1}=(1 / 4)\left[\left(1-3 x^{2}\right)^{2}+3\left(1-x^{2}\right)^{2} \cos ^{2} 2 \varphi\right] ; f=\left(1-x^{2}\right)^{2} .
\end{aligned}
$$


These equations were solved numerically[4, 11] and the first equations appear to give either Goldstone modes or modes with vanishing energies (of order $0.03 \Delta_{0}(T)-0.08 \Delta_{0}(T)$ ), while five high-frequency modes in each state were obtained from the second equations.

We shall now present results for high-frequency modes ( $E_{i}$ is the energy (frequency) of the $i$ th branch).

$$
\begin{aligned}
& E_{1,2}=\Delta_{0}(T)(1.93-i 0.41) ; \\
& E_{3}=\Delta_{0}(T)(1.62-i 0.75) ; \\
& E_{4,5}=\Delta_{0}(T)(1.59-i 0.83)
\end{aligned}
$$

Comparison of these results with spectrum of pure $d_{x-y}{ }^{2}{ }^{2}$ and $d_{x y}$-states, obtained by Brusov et al. as follows: $[4,11]$

$$
\begin{aligned}
& E_{1}=\Delta_{0}(T)(1.88-i 0.79) ; \\
& E_{2}=\Delta_{0}(T)(1.66-i 0.50) ; \\
& E_{3}=\Delta_{0}(T)(1.40-i 0.68) ; \\
& E_{4}=\Delta_{0}(T)(1.13-i 0.71) ; \\
& E_{5}=\Delta_{0}(T)(1.10-i 0.65)
\end{aligned}
$$

These results led them to conclusion that in spite of the fact that spectra in both pure states, $d_{x-y}^{2}{ }^{2}$ and $d_{x y}$, turn out to be identical, the spectrum in mixed $d_{x-y}{ }^{2}{ }^{2}$ - and $d_{x y}$-state is quite different from that in pure states. All modes are non-degenerated in pure states, but two high-frequency modes are twice degenerated in mixed state. The collective modes have higher frequencies in mixed state - from $1.59 \Delta_{0}(T)$ up to $1.93 \Delta_{0}(T)$, compared to $1.1 \Delta_{0}(T)$ up to $1.88 \Delta_{0}(T)$ in pure state. In pure d-wave states, damping of collective modes is also bigger than in mixed state (in pure states $\operatorname{Im} E_{i}$ is from $30 \%$ to $65 \%$ and in mixed state from $20 \%$ to $50 \%$ ). This is because the gap vanishes in pure states along chosen lines while it vanishes just at two points (poles) in mixed state. [4]

Such difference in the spectra of collective modes in pure $d$-wave states and in mixed $d$-wave state enables us to probe the symmetry of states using ultrasound and/or microwave absorption experiments. These experiments require high frequencies (in the order of tens of $\mathrm{GHz}$ as in the experiment of Feller et al. [16]), but there are no principle restrictions for ultrasound (microwave) frequencies: frequencies of collective modes are proportional to gap $\Delta_{0}(T)$, and the gap vanishes at $T_{c^{\prime}}$ so one could in principle use any frequency approaching $T_{c}$.

Note that the case of the $d_{x-y}^{2}{ }^{2}+i d_{x y}$-state was also considered by Balatsky et al., [17] who studied one of the possible collective modes in this state. They considered a superconducting state with mixed-symmetry-order parameter components, e.g., $d+i$ s and $d_{x-y}^{2}{ }^{2}+i d_{x y}$ and argued for the existence of a new orbital magnetization mode which corresponds to oscillations of relative 
phase $\varphi$ between two components around an equilibrium value of $\varphi=\pi / 2$. The analogue of this mode is the so-called clapping mode of the superfluid A-phase in ${ }^{3} \mathrm{He}$. The frequency of this mode $\omega_{0}(B, T)$ depending on the field and temperature for the specific case of magneticfield-induced $d_{x y}$-state has been estimated. This mode is tunable with a magnetic field with $\omega_{0}(B, T) \infty B \Delta_{0}$, where $\Delta_{0}$ is the amplitude of gap of the order parameter in $d$-wave state. The velocity $s(B, T)$ of this mode has been estimated as well.

\section{4. $d_{x-y}^{2}{ }^{2}$-state of high-temperature superconductors with a small admixture of $d_{x y}$-state}

The whole spectrum of collective modes in mixed $d_{x-y}{ }^{2}+i \varepsilon d_{x y}$ state of HTSC with arbitrary admixture of $d_{x y}$-state is determined by the above equations (17). For interpretation of the sound attenuation and microwave absorption data as well as for identification of the type of pairing and order parameter in unconventional superconductors knowledge of the collective-mode spectrum could be used. In particular, these experiments allow the estimation in a possible mixed state of the extent of admixture of a $d_{x y}$-state.

The case of small $\gamma$ is, however, the most interesting case: one supposes that $d_{x}^{2}-y^{2}$-state is the dominant state and the admixture of $d_{x y}$-state is small, say 3-10\%. For this case one can expand all expressions in powers of small $\gamma$ and obtain the corrections to the equations for the spectrum of pure $d_{x-y}^{2}{ }^{2}$-state, which has been found before [11, 13].

Let us consider the case of small admixture of $d_{x y}$-state (small $\varepsilon$ ): we suppose that dominant state is $d_{x-y}^{2}{ }^{2}$-state and admixture of $d_{x y}$-state is small, about 3-10\%. After expanding of all expressions in powers of small $\varepsilon$ we obtain the below corrections.

We use the following notations:

$$
\begin{aligned}
& a=\left(1-x^{2}\right)^{2} \cos ^{2} 2 \phi ; \\
& b=\left(1-x^{2}\right)^{2} \sin ^{2} 2 \phi ; \\
& a_{1}=a+\omega^{2},
\end{aligned}
$$

Here, we obtain the following expressions:

$$
\begin{gathered}
\sqrt{\omega^{2}+4\left(1-x^{2}\right)^{2}\left[\cos ^{2} 2 \phi+\gamma^{2} \sin ^{2} 2 \phi\right]} \approx \sqrt{a_{1}}\left(1+\gamma^{2} \frac{b}{2 a_{1}}\right) \\
\ln f \approx \ln a+\gamma^{2} \frac{b}{a} \\
\frac{\sqrt{\omega^{2}+4\left(1-x^{2}\right)^{2}\left[\cos ^{2} 2 \varphi+\gamma^{2} \sin ^{2} 2 \varphi\right]}+\omega}{\sqrt{\omega^{2}+4\left(1-x^{2}\right)^{2}\left[\cos ^{2} 2 \varphi+\gamma^{2} \sin ^{2} 2 \varphi\right]}-\omega} \approx A+\gamma^{2} B,
\end{gathered}
$$


where

$$
\begin{gathered}
A=\frac{\left(\sqrt{a_{1}}+\omega\right)^{2}}{a} ; \\
B=\frac{b}{a^{2}} \frac{\sqrt{a_{1}}+\omega}{\sqrt{a_{1}}}\left[a+\sqrt{a_{1}}\left(\omega+\sqrt{a_{1}}\right)\right] \\
\ln \frac{\sqrt{\omega^{2}+4\left(1-x^{2}\right)^{2}\left[\cos ^{2} 2 \phi+\gamma^{2} \sin ^{2} 2 \varphi\right]}+\omega}{\sqrt{\omega^{2}+4\left(1-x^{2}\right)^{2}\left[\cos ^{2} 2 \phi+\gamma^{2} \sin ^{2} 2 \varphi\right]}-\omega} \approx \\
\approx \ln \frac{\sqrt{\omega^{2}+4\left(1-x^{2}\right)^{2} \cos ^{2} 2 \phi}+\omega}{\sqrt{\omega^{2}+4\left(1-x^{2}\right)^{2} \cos ^{2} 2 \phi}-\omega}+\gamma^{2} C
\end{gathered}
$$

where

$$
C=\frac{b\left(\sqrt{a_{1}}+\omega\right)}{\sqrt{a}}+\frac{b}{a} .
$$

For other expressions, we obtain, with the accuracy of order $\gamma^{2}$ :

$$
\begin{aligned}
& \frac{\sqrt{\omega^{2}+4\left(1-x^{2}\right)^{2}\left[\cos ^{2} 2 \varphi+\gamma^{2} \sin ^{2} 2 \varphi\right]}}{\omega} \times \\
& \times \ln \frac{\sqrt{\omega^{2}+4\left(1-x^{2}\right)^{2}\left[\cos ^{2} 2 \varphi+\gamma^{2} \sin ^{2} 2 \varphi\right]}+\omega}{\sqrt{\omega^{2}+4\left(1-x^{2}\right)^{2}\left[\cos ^{2} 2 \varphi+\gamma^{2} \sin ^{2} 2 \varphi\right]}-\omega} \approx \\
& \frac{\sqrt{\omega^{2}+4\left(1-x^{2}\right)^{2} \cos ^{2} 2 \varphi}}{\ln \frac{\sqrt{\omega^{2}+4\left(1-x^{2}\right)^{2} \cos ^{2} 2 \varphi}}{\sqrt{\omega^{2}+4\left(1-x^{2}\right)^{2}} \cos ^{2} 2 \varphi}-\omega}+ \\
& +\frac{\gamma^{2}}{\omega} \sqrt{a_{1}}\left(C+\frac{b}{2 a_{1}} \ln \frac{\sqrt{\omega^{2}+4\left(1-x^{2}\right)^{2} \cos ^{2} 2 \varphi}+\omega}{\sqrt{\omega^{2}+4\left(1-x^{2}\right)^{2} \cos ^{2} 2 \varphi}-\omega}\right)
\end{aligned}
$$




$$
\begin{aligned}
& \frac{\omega}{\sqrt{\omega^{2}+4\left(1-x^{2}\right)^{2}\left[\cos ^{2} 2 \phi+\gamma^{2} \sin ^{2} 2 \phi\right]}} \times \\
& \times \ln \frac{\sqrt{\omega^{2}+4\left(1-x^{2}\right)^{2}\left[\cos ^{2} 2 \phi+\gamma^{2} \sin ^{2} 2 \phi\right]}+\omega}{\sqrt{\omega^{2}+4\left(1-x^{2}\right)^{2}\left[\cos ^{2} 2 \phi+\gamma^{2} \sin ^{2} 2 \phi\right]}-\omega} \approx \\
& \frac{\omega}{\sqrt{\omega^{2}+4\left(1-x^{2}\right)^{2} \cos ^{2} 2 \phi+} \ln \frac{\sqrt{\omega^{2}+4\left(1-x^{2}\right)^{2} \cos ^{2} 2 \phi}+\omega}{\sqrt{\omega^{2}+4\left(1-x^{2}\right)^{2}} \cos ^{2} 2 \phi}-\omega}+ \\
& \left.+\gamma^{2} \frac{\omega}{\sqrt{a_{1}}\left(C-\frac{b}{2 a_{1}} \ln \frac{\sqrt{\omega^{2}+4\left(1-x^{2}\right)^{2} \cos ^{2} 2 \phi}}{\sqrt{\omega^{2}+4\left(1-x^{2}\right)^{2} \cos ^{2} 2 \phi}}-\omega\right.}\right) .
\end{aligned}
$$

Putting all expressions (23)-(31) into (17) one obtains the whole set of equations for the collective-mode spectrum of mixed $d_{x-y}^{2}{ }^{2}+i \varepsilon d_{x y}$ state with small admixture of $d_{x y}$-state to $d_{x-y}^{2}{ }^{2}$-state.

\section{Conclusions}

In order to solve one of the problems of unconventional superconductivity - the exact form of the order parameter - we consider the mixed $(1-\gamma) d_{x-y}{ }^{2}{ }^{2}+i \gamma d_{x y}$ state in HTSC. A full set of equations for the collective-modes spectrum in a mixed d-wave state with an arbitrary admixture of $d_{x y}$-state has been derived. These equations have been solved for the case of equal admixtures $d_{x-y}^{2}{ }^{2}$ - and $d_{x y}$-states by Brusov et al. [4, 11, 14] It has been shown that the difference of collective excitations spectrum in mixed $d$-wave states and in pure state provide the possibility of probing the symmetry of the state by ultrasound and/or microwave absorption experiments.

The case of small $\gamma$ is the most interesting case: one supposes that $d_{\mathrm{x}-\mathrm{y}}{ }^{2}$-state is the dominant state and admixture of $d_{x y}$-state is small, say 3-10\%. In this case all expressions can be expanded in powers of small $\gamma$ and the corrections to the spectrum of pure $d_{x-y}{ }^{2}$-state, which has been found before, can be obtained. [18]

For identification of the type of pairing and determination of the exact form of the order parameter in unconventional superconductors the results obtained could be quite useful. They allow evaluation of the extent of an admixture of a $\mathrm{d}_{\mathrm{xy}}$-state in relation to the spectrum of pure $\mathrm{d}_{\mathrm{x}-\mathrm{y}}^{2}$-state in a possible mixed state. Obtained equations allow the calculation of the whole collective mode. This spectrum could be used for interpretation of the sound attenuation and microwave absorption data. [18]

The results obtained could also allow three quite important questions to be answered: [18] 
1. Does the gap disappear along some chosen lines?

2. Is there a pure or mixed d-wave state in HTSC?

3. How huge is the admixture of $d_{x y}$-state to $\mathrm{d}_{x 2-\mathrm{y} 2}$-state in a possible mixed state?

\section{Author details}

Peter Brusov* and Tatiana Filatova

*Address all correspondence to: pnb1983@yahoo.com

Financial University under the Government of Russian Federation, Russia

\section{References}

[1] Krishana, K. et al. (1997), Science 277, 83.

[2] Annett, J. F., Goldenfeld, N. D. and Leggett, A. J. (1996), In: Physical Properties of High Temperature Superconductors V, ed. D. M. Ginsberg (World Scientific, Singapore).

[3] Laughlin, R. B. (1998), Phys. Rev. Lett. 80, 5188.

[4] Brusov, P., and P. Brusov (2000), Physica B, 281\&282, 949.

[5] Brusov, P. N., Brusova, N. P. (1994), Physica B 194-196, 1479.

[6] Brusov. P. N., Brusova, N. P. (1996), J. Low Temp. Phys., 101, 1003.

[7] Brusov, P. N., Brusova, N. P. and Brusov, P. P. (1996), Czechoslovak Journal of Physics, 46, suppl. s2, 1041.

[8] Brusov, P. N., Brusova, N. P. Brusov, P.P. (1997), J. Low Temp. Phys. 108, 143; Physica B 259-261, 496 (1999).

[9] Brusov, P. N., Brusova, N. P., Brusov, P. P., Harabaev, N. N. (1997), Physica C $282-$ $287,1881$.

[10] Brusov, P. N., Brusova, N. P., Brusov, P. P. (1996), Fiz. Nizk. Temp. 22, 506 [Low Temp. Phys., 22, 389].

[11] Brusov, P. N. (1999), Mechanisms of High Temperature Superconductivity, v.1, 2; Rostov State University Publishing, p. 1389. 
[12] Brusov, P. N., Brusov, P.P. (2001), “Collective properties of superconductors with nontrivial pairing", Sov. Phys. JETP, 119, 913-930 [Zh. Eksp. Theor. Phys. v.92, p. 795].

[13] Brusov, P. N., Brusova, N. P. and Brusov, P. P. (1997), The collective excitations of the order parameter in HTSC and heavy fermion superconductors (HFSC) under d-pairing. J. Low Temp. Phys. 108, 143.

[14] Brusov P., Brusov, Majumdar, P. and Orehova, N. (2003), “Ultrasound attenuation and collective modes in mixed $d_{\mathrm{x} 2 \mathrm{-y} 2}+d_{\mathrm{xy}}$ state of unconventional superconductors", Brazilian Journal of Physics, 33, 729-732.

[15] Brusov, P., Brusov, P. and Lee, C. (2004), Collective properties of unconventional superconductors, Int. J. Mod. Phys. B, v.18, 867-882.

[16] Feller J. R., Tsai, C. C., Ketterson, J. B. et al. (2002), Phys. Rev. Lett. 88, 247005.

[17] Balatsky, A. V., Kumar, P. and Schrieffer, J. R. (2000), Phys. Rev. Lett. 84, 4445.

[18] Brusov, P., Brusov, P. (2009) Collective Excitations in Unconventional Superconductors and Superfluids, pp. 860, World Scientific Publishing, Singapore 